\title{
PeerCare: Challenging the Monitoring Approach to Eldercare
}

\author{
Yann Riche \\ INRIA/LRI, Univ. Paris-Sud, France - Univ. of Queensland, Australia \\ yann.riche@lri.fr
}

This Ph.D. is jointly advised by Wendy Mackay (INRIA) and Stephen Viller (Univ. of Queensland). It takes place in the Human Computer Interaction and Computer Mediated Communications research areas, and focuses on the design and evaluation of communcation appliances for seniors to better "age in place".

\section{Introduction}

Recent projections show that by $2050,34 \%$ of the European population will be aged 65 or more. Institutions are seeking alternatives to existing care solutions, focusing essentially on providing support for the elderly to stay at home. To date, most of the related research has been focused towards monitoring technologies to overcome this issue. While these systems are a mean to help the caregiver, they only indirectly help the elderly. Moreover, using automatons as caregivers presents many problems [1], including stigmatization of dependance. In contrast, studies like Giles et al.'s [2] emphasize the role of social networks well-being of elderly. Therefore, alternatives are needed for independent people with no critical health concerns who are willing to be proactive about their health and to maintain high social connectedness. My project adopts this approach by seeking ways of supporting informal care behaviours to help the elderly "age in place". My primary focus is to explore how communication appliances can play a significant role in this regard. My work also seeks to validate designs and concepts by conducting user-centered and participatory design processes, and by validating them using in situ, longtudinal deployments. Communication appliances, introduced in the interLiving project [3], are defined as a "[...]simple-to-use, single-function devices that let people communicate, passively or actively, via some medium, with one or more remotely-located friends or family" 44. Мy project seeks to benefit from the existing social networks of elderly and use communication appliances to strenghthen their role in elderly's well aging.

\section{Research Methodology}

Communication appliances is a fairly new concept and I believe it has a vast potential to help seniors age in place. The goal of this $\mathrm{PhD}$ is to explore this potential and provide seniors with prototypes and devices they will use. For

C. Baranauskas et al. (Eds.): INTERACT 2007, LNCS 4663, Part II, pp. 628 630, 2007.

(C) IFIP International Federation for Information Processing 2007 
this purpose, my research methodology is to Use participatory design and user-centered design techniques to better understand elderly people's needs and how communication appliances can be smoothly integrated to their environment, Implement a technology probe to study the communication in situ and bring the design process into their environment, and Design, prototype and deploy several communication appliances with seniors to support their communication.

\section{$3 \quad$ PeerCare}

To understand the role of communication for seniors living at home, I conducted a user study with women aged between 62 and 88, living in private flat or in sheltered housing. The major outcome of this study was the important role of seniors' relationships with each other in their well being. The concept of PeerCare, central line in this $\mathrm{PhD}$, is defined as senior to senior relationships in which people provide informal reciprocal care to one another. This opposes the current monitoring approaches where the relationship is unilateral. The particularity of PeerCare behaviors is that it exists between people sharing a common interest in having someone care for them. The intensity, frequency and type of care may vary over time, but the flow of care is overly maintained and bilateral.

Several major questions are raised for the design, development and evaluation of technology to support PeerCare: How to overcome technical problems to deploy the technology in situ? How to approach, motivate and imply users in the design? How to objectively evaluate the benefit of a communication appliance? These questions raises fondamental issues concerning the approach to integrate research technologies in a home environment, recrute users and evaluate communications/ambient devices. To answer the first question, I have designed and implemented markerClock [5], a device designed to study the benefit of communication appliances for elderly. To explore the second question, I have a conducted a participatory design process looking at communication devices for elderly in general and PeerCare in particular. To provide answers to the third question related to the evaluation of communication appliances and their benefits, I will deploy and evaluate markerClock in elderly people's homes.

\section{References}

[1] Haigh, K.Z., Yanco, H.A.: Automation as caregiver: A survey of issues and technologies. In: AAAI Workshop Automation as Caregiver, pp. 39-53 (2002)

[2] Giles, L., Glonek, G., Luszcz, M., Andrews, G.: Effects of social networks on 10 year survival in very old Australians: The Australian longitudinal study of aging. Journal of Epidemiology and Community Health 59, 574-579 (2005)

[3] Hutchinson, H., Mackay, W., Westerlund, B., Bederson, B.B., Druin, A., Plaisant, C., Beaudouin-Lafon, M., Conversy, S., Evans, H., Hansen, H., Roussel, N., Eiderback, B.: Technology probes: inspiring design for and with families. In: Conf. on Human factors in Computing Systems, Ft. Lauderdale, USA, pp. 17-24. ACM Press, New York (2003) 
[4] Mackay, W., Riche, Y., Labrune, J.-B.: Communication appliances: Shared awareness for intimate social networks. In: ACM SIGCHI 2005 Workshop on Awareness Systems: Known results, theory, concepts and future challenges, Portland, OR, US, ACM Press, New York (2005)

[5] Riche, Y., Mackay, W.: Markerclock: A communicating augmented clock for elderly [short paper under review]. In: Baranauskas, C., Palanque, P., Abascal, J., Barbosa, S.D.J. (eds.) Interact 2007. LNCS, vol. 4663, Springer, Heidelberg (2007) 